\title{
Diagnosis of Jejunal Metastases from Lung Cancer Using Capsule Endoscopy
}

\author{
Charlotte Leduc $^{\mathrm{a}} \quad$ Nathalie Prim $^{\mathrm{a}} \quad$ Bertrand Mennecier $^{\mathrm{a}}$ \\ Michel Delvaux ${ }^{b}$ Afshin Gangi ${ }^{c}$ Elisabeth Quoix ${ }^{a}$
}

${ }^{a}$ Service de pneumologie, Pôle de Pathologie Thoracique, Nouvel Hôpital Civil, Hôpitaux Universitaires de Strasbourg, Strasbourg, France; ${ }^{b}$ Service de gastro-entérologie, Nouvel Hôpital Civil, Hôpitaux Universitaires de Strasbourg, Strasbourg, France; 'Service d'imagerie interventionnelle oncologique et viscérale, Nouvel Hôpital Civil, Hôpitaux Universitaires de Strasbourg, Strasbourg, France

\section{Keywords}

Jejunal metastasis · Lung cancer · Capsule endoscopy

\begin{abstract}
Gastrointestinal metastases from lung cancer are rare and usually asymptomatic. We report a case of small bowel metastases from primary lung cancer revealed by abdominal pain and severe recurrent anaemia. The diagnosis was obtained with capsule endoscopy. This noninvasive procedure thus represents a valuable method contributing to a rapid and detailed diagnosis while reducing underdiagnosis, and it should thus be considered for lung cancer patients complaining of abdominal symptoms, which may indeed be related to gastrointestinal metastases.

(C) 2016 The Author(s) Published by S. Karger AG, Basel
\end{abstract}

\section{Introduction}

About $50 \%$ of patients with a diagnosis of non-small cell lung carcinoma exhibit metastatic disease at the time of diagnosis. The preferential sites of extrapulmonary spread are 


\section{Case Reports in Oncology}

the lymph nodes, liver, brain, adrenal glands, and bones. Gastrointestinal metastases are rare and usually asymptomatic.

\section{Case Report}

A 51-year-old smoker was diagnosed with a poorly differentiated non-small cell lung carcinoma of the right lower lobe in stage cT2a N0 M1b (brain metastases). He received front-line therapy with cisplatin-pemetrexed. A CT scan after 4 cycles revealed a partial response in the lung and a complete response in the brain. Maintenance therapy with pemetrexed was administered. After 2 cycles, locoregional progression was observed, with the extrapulmonary disease still controlled. The chemotherapy was switched to paclitaxel. After 2 cycles, the patient complained of abdominal pain and reported melena, yet without signs of intestinal obstruction. Biological data revealed severe aregenerative anaemia (haemoglobin concentration at $6.9 \mathrm{~g} / \mathrm{dl}$, reticulocyte count at $120,000 / \mathrm{mm}^{3}$ ) and iron deficiency signs consisting of low serum ferritin, iron, and transferrin saturation. The stool examination for occult blood was strongly positive, while gastric fiberscopy and colonoscopy results were normal. Abdominal CT with contrast medium enhancement showed an intussusception of the small bowel (fig. 1). We then decided to explore the small bowel by means of capsule endoscopy, which revealed several secondary lesions, including one stenosing the first jejunal loop and another one ulcerated and bleeding (fig. 2; online suppl. videos 1 and 2; for all online suppl. material, see www.karger.com/doi/10.1159/000447349). A double-balloon enteroscopy was performed thereafter for lesion biopsy, confirming the metastatic localisation of a poorly differentiated lung carcinoma. On account of the patient's poor performance status, surgical treatment was not considered, with only symptomatic treatment performed using several blood transfusions and iron supplementation. At last, the patient died 20 months following the cancer diagnosis and 5 months after discovering the intestinal metastasis.

\section{Discussion}

Improvement in metastatic lung cancer management has led to extending survival, and we are thus faced with an increasing number of uncommon metastatic sites. Gastrointestinal metastases are considered to be rare, usually occurring in advanced disease stages, and associated with poor prognosis [1]. Due to their mostly asymptomatic presentation, these lesions are probably underdiagnosed. In the most recent autopsy series, gastrointestinal metastases were observed in $12 \%$ of patients [2], with the small bowel as the most common site of metastatic involvement. Gastric and duodenal metastases likely cause abdominal pain, haematemesis, as well as chronic bleeding resulting in anaemia. Small bowel involvement often leads to acute abdomen, and may be associated with various clinical complications such as perforation, obstruction, or haemorrhage [3]. Clinical cases of gastrointestinal metastases from lung cancer have been reported, with diagnosis systematically delayed [4]. As illustrated in our case report, although conventional imaging revealed jejunal intussusception, the correct diagnosis of metastatic intestinal involvement was only brought upon by capsule endoscopy. This non-invasive procedure thus represents a valuable method contributing to a rapid and detailed diagnosis while reducing underdiagnosis [5]. Treatment consists in promptly performing exploratory laparotomy. Based on published data, this strategy 


\section{Case Reports in Oncology}

Leduc et al.: Diagnosis of Jejunal Metastases from Lung Cancer Using Capsule Endoscopy

allows for obtaining a therapeutic effect in patients with solitary gastrointestinal metastases, and a favourable palliative effect on survival in those with preoperatively diagnosed metastases.

\section{Conclusion}

We reported a case of small bowel metastases from primary lung cancer revealed by abdominal pain and severe recurrent anaemia. Capsule endoscopy allowed for an aetiologic diagnosis to be obtained, and should thus be considered in lung cancer patients complaining of abdominal symptoms, which may indeed be related to gastrointestinal metastases.

\section{Statement of Ethics}

Written informed consent was obtained from the patient for publication of this case report and any accompanying images.

\section{Disclosure Statement}

The author(s) indicate no potential conflicts of interest.

\section{References}

1 Yang CJ, Hwang JJ, Kang WY, et al: Gastro-intestinal metastasis of primary lung carcinoma: clinical presentations and outcome. Lung Cancer 2006;54:319-323.

-2 Yoshimoto A, Kasahara K, Kawashima A: Gastrointestinal metastases from primary lung cancer. Eur J Cancer 2006;42:3157-3160.

3 Rossi G, Marchioni A, Romagnani E, et al: Primary lung cancer presenting with gastrointestinal tract involvement: clinicopathologic and immunohistochemical features in a series of 18 consecutive cases. J Thorac Oncol 2007;2:115-120.

4 Hillenbrand A, Sträter J, Henne-Bruns D: Frequency, symptoms and outcome of intestinal metastases of bronchopulmonary cancer. Case report and review of the literature. Int Semin Surg Oncol 2005;2:13.

5 Rondonotti E, Pennazio M, Toth E, et al: Small-bowel neoplasms in patients undergoing video capsule endoscopy: a multicenter European study. Endoscopy 2008;40:488-495. 


\section{Case Reports in Oncology}

\begin{tabular}{l|l}
\hline Case Rep Oncol 2016;9:526-529 \\
\hline DOI: 10.1159/000447349 & $\begin{array}{l}\text { C 2 2016 The Author(s). Published by S. Karger AG, Basel } \\
\text { www.karger.com/cro }\end{array}$ \\
\hline
\end{tabular}

Leduc et al.: Diagnosis of Jejunal Metastases from Lung Cancer Using Capsule Endoscopy
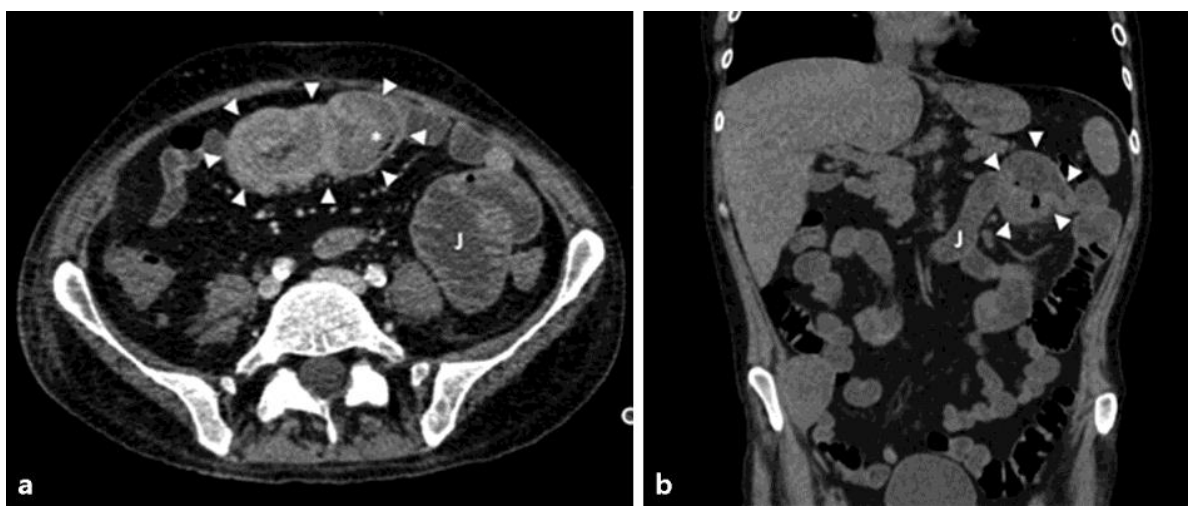

Fig. 1. CT images. a Axial section showing the intussusception of the small bowel (target-like) caused by the jejunal metastasis (arrowheads). The air in the wall represents necrosis $(*)$. The downstream jejunum (J) is dilated and stripy. b Coronal section showing the intussusception (arrowheads), with stasis in the top part and the fleshy tumour in the bottom part. The small residual lumen is indicative of slowed intestinal transit. J: normal jejunum.
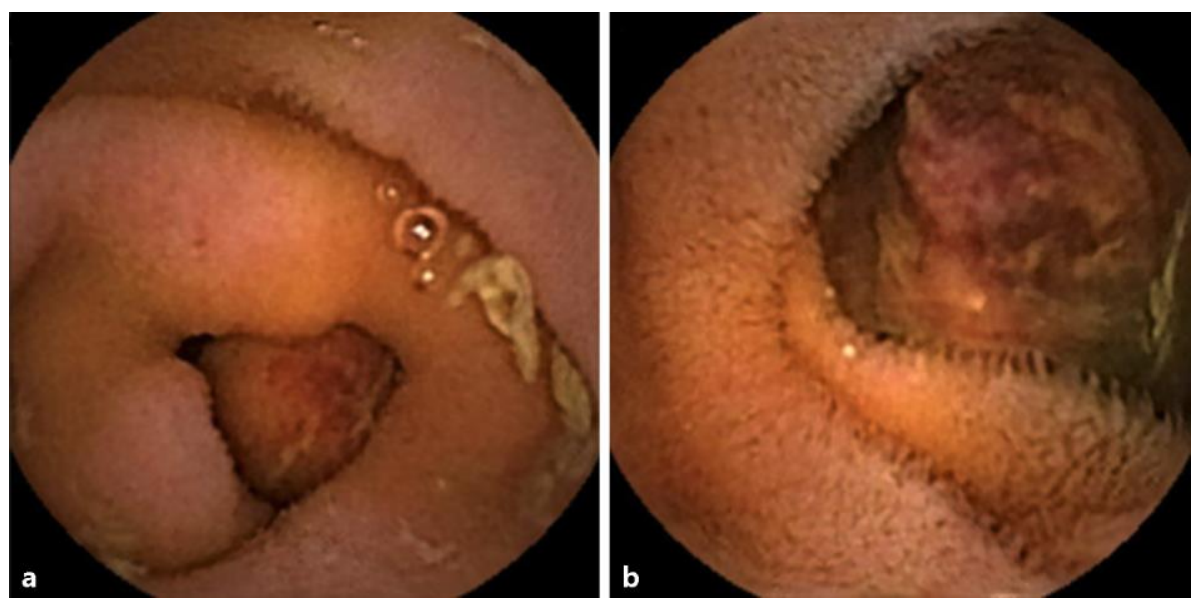

Fig. 2. Capsule endoscopy. a Jejunal metastasis obstructing the first intestinal loop. b Ulcerated tumour, at the origin of bleeding. 